\title{
Surgical repair of post-infarction ventricular free-wall rupture in the Netherlands: data from a nationwide registry
}

\author{
Matteo Matteucci ${ }^{1,2 \#}$, Daniele Ronco ${ }^{1,2 \#}$, Justine M. Ravaux ${ }^{1}$, Giulio Massimi ${ }^{1,2}$, Michele Di Mauro ${ }^{1}$, \\ Saskia Houterman $^{3}$, Jos Maessen ${ }^{1,4}$, Cesare Beghi ${ }^{2}$, Paolo Severgnini ${ }^{5}$, Roberto Lorusso ${ }^{1,4}$; on behalf of \\ the Cardiothoracic Surgery Registration Committee of the Netherlands Heart Registration*
}

${ }^{1}$ Department of Cardiothoracic Surgery, Heart and Vascular Centre, Maastricht University Medical Centre, Maastricht, The Netherlands; ${ }^{2}$ Department of Surgical and Morphological Sciences, Circolo Hospital, University of Insubria, Varese, Italy; ${ }^{3}$ Netherlands Heart Registration, Utrecht, The Netherlands; ${ }^{4}$ Cardiovascular Research Institute Maastricht (CARIM), Maastricht, The Netherlands; ${ }^{5}$ Department of Biotechnology and Sciences of Life, University of Insubria, Varese, Italy

\#These authors contributed equally to this work.

Correspondence to: Matteo Matteucci, MD. Department of Cardiothoracic Surgery, Heart and Vascular Centre, Maastricht University Medical Centre (MUMC), P. Debyelaan 12, 6221 AZ Maastricht, The Netherlands. Email: matteomatteucci87@gmail.com.

\begin{abstract}
Background: Ventricular free-wall rupture (VFWR) is an infrequent but catastrophic complication of acute myocardial infarction (AMI). Most reports about outcome after surgical treatment are single-center experiences. We examined the early and mid-term outcomes after surgical repair of post-AMI VFWR using the Netherlands Heart Registration (NHR) database.

Methods: We included data from NHR patients (>18 years old) who underwent surgery for post-AMI VFWR between 2014 and 2019. The primary end-point was in-hospital mortality. Secondary outcomes included postoperative complications and mid-term survival.

Results: The study included 148 patients (54.7\% male, mean age $66.5 \pm 11.1$ years). Critical preoperative status was found in $62.6 \%$ of subjects. In-hospital mortality was $31.1 \%$ (46 of 148). Multivariable analysis identified female sex [odds ratio (OR), 5.49; $95 \%$ confidence interval (CI): 2.24-13.46] and critical preoperative status (OR, 4.06; 95\% CI: 1.36-12.13) as independent predictors of in-hospital mortality. The overall median postoperative follow-up was 2.2 (interquartile range, 0.7-3.8) years. Overall survival rates at three and five years were $58.9 \%$ and $55.7 \%$, respectively. Among hospital survivors, only 15 (14.7\%) patients died during follow-up, with a five-year survival rate of $80.8 \%$.

Conclusions: In-hospital mortality after surgical repair of post-AMI VFWR is considerable. Female sex and preoperative critical status are independent predictors of early postoperative (in-hospital) death. Logistic EuroSCORE I can reliably predict in-hospital mortality (optimal cut-off $>33 \%$ ). Mid-term follow-up of patients surviving in-hospital course shows excellent results.
\end{abstract}

Keywords: Cardiac rupture; ventricular free-wall rupture (VFWR); myocardial infarction (MI); surgical repair

Submitted Apr 16, 2021. Accepted for publication Oct 20, 2021.

doi: 10.21037/acs-2021-ami-10

View this article at: https://dx.doi.org/10.21037/acs-2021-ami-10

\footnotetext{
* Members of the Cardiothoracic Surgery Registration Committee: S Bramer (Amphia); WJP van Boven (Amsterdam UMC, locatie AMC); ABA Vonk (Amsterdam UMC, locatie VUmc); BMJA Koene (Catharina Ziekenhuis); JA Bekkers (Erasmus MC); GJF Hoohenkerk (HagaZiekenhuis); ALP Markou (Isala); A de Weger (Leids Universitair Medisch Centrum); P Segers (Maastricht UMC+); F Porta (Medisch Centrum Leeuwarden); RGH Speekenbrink (Medisch Spectrum Twente); W Stooker (OLVG); WWL Li (Radboudumc); EJ Daeter (St. Antonius Ziekenhuis); NP van der Kaaij (UMC Utrecht); G Vigano (Universitair Medisch Centrum Groningen).
} 


\section{Introduction}

The development of a post-acute myocardial infarction (AMI) ventricular free-wall rupture (VFWR) is an uncommon but highly fatal complication, occurring in less than $1 \%$ of patients sustaining AMI in the modern era of early reperfusion therapy (1). In post-AMI VFWR patients treated medically, mortality rate accounts for approximately $90 \%$ (2), whereas mortality in patients undergoing surgical repair ranges between $17 \%$ and $36 \%$ (3-5). Given the rare nature of this entity, most previous studies on surgical outcomes have been confined to single-center retrospective analyses with a relatively small sample size.

The aim of the present study was, therefore, to utilize a nationwide registry to provide contemporary results of the surgical treatment for post-AMI VFWR. Furthermore, we aim to identify factors that predict the risk for poor outcomes after surgery.

\section{Methods}

\section{Study design and outcomes}

This is a retrospective cohort study in which data from the Netherlands Heart Registration (NHR) was analyzed. The NHR is a Dutch nationwide, physician-driven and patientfocused quality registry initiated in 2017 and merges three national registries (6). It contains procedural and outcome data of various cardiac interventions, including surgery, from the 16 hospitals performing cardiac surgery in the Netherlands. Data collection regarding daily activity is mandatory and submitted for audit; registration is accomplished by the participating centers in a secure online environment and transferred in an encrypted format to a central server. In-hospital and post-discharge data are registered, including survival status and any rehospitalization or re-intervention required during active follow-up (one to five years after surgery).

In the current study, we included adults (age $>18$ years) undergoing surgical repair of a post-AMI VFWR between January 1, 2014 and December 31, 2019. Eligible individuals were patients who underwent repair of cardiac rupture as a consequence of AMI, including patients operated either for VFWR alone, or for VFWR combined with ventricular septal rupture (VSR).

Given the nature of the NHR, the need for informed consent from each patient was waived. The research protocol of the current study was approved by the NHR
Scientific Board. The anonymized data was used in accordance with the Declaration of Helsinki principles.

\section{Definitions}

The definitions are in accordance with the NHR Handbook (version 2020), available via the website (http://www. nederlandehartregistratioe.nl/). The primary outcome of this analysis was in-hospital (early) mortality; additional outcomes were postoperative complications, predictors of in-hospital mortality and mid-term survival. Mortality was assessed by the hospitals using the national Personal Records Database (BRP).

Several variables were included in this study. Unstable angina was defined as chest pain requiring intravenous nitrate infusion up to the time of surgery. Critical preoperative status included any of the following conditions: cardiac arrest before the operation, need for mechanical ventilation, inotropic support, intra-aortic balloon pump (IABP) before transfer to the operating room, or preoperative renal failure (i.e., anuria or oliguria $<10 \mathrm{~mL} / \mathrm{h}$ ). Perioperative AMI was identified according to the definition by the Society of Thoracic Surgeons (7). Postoperative renal failure was defined as the need for any renal replacement therapy, or increase in serum creatinine $>177 \mu \mathrm{mol} / \mathrm{L}$ or double the preoperative value. Re-thoracotomy within 30 days included any reoperation performed, excluding procedures related to mediastinitis or sternal resynthesis.

\section{Statistical analysis}

Continuous variables have been tested for normality distribution with the Shapiro-Wilk test and are reported as mean \pm standard deviation (variables not violating the normality assumption) or median with interquartile range (IQR) (variables violating the normality assumption). Categorical variables are presented as frequencies with percentages. After the analysis of patterns, multiple imputation using an automatic method was performed for missing values. Only variables with less than $40 \%$ of data missing were included in the analysis (8). Continuous variables were compared individually (univariate analysis) with the Student's $t$-test or the Mann-Whitney U-test, and categorical variables were tested with the Chi-square or Fisher's exact test, as appropriate. Variables of clinical interest that achieved a $\mathrm{P}$ value $<0.10$ in the univariate analysis were tested for multicollinearity and then entered 
into a multivariable logistic regression analysis to identify independent predictors of in-hospital mortality. Receiver operating characteristic (ROC) curves were calculated for strongly significant continuous variables to determine the optimal cut-off value for predicting in-hospital mortality; the point with the largest sum of sensitivity and specificity was chosen as the threshold. Survival curves were constructed with the Kaplan-Meier method for both the whole population and hospital survivors only, with a subgroup analysis according to sex, and compared using the log-rank test. All statistical analyses were conducted using SPSS 26.0 (IBM Corp. Released 2019. IBM SPSS Statistics for Windows, Version 26.0. Armonk, NY, USA). A twotailed $\mathrm{P}$ value $<0.05$ was considered statistically significant.

\section{Results}

\section{Clinical characteristics}

The overall cohort consisted of 148 patients (81 male, $54.7 \%$ ) with an average age of $66.5 \pm 11.1$ years. Imputed baseline and perioperative characteristics tested for potential inclusion into the multivariable model are presented in Table 1, and raw baseline data (before imputation) are shown in Tables S1,S2. Common major comorbidities included diabetes mellitus (12.8\%), chronic obstructive pulmonary disease (COPD) (10.8\%), peripheral vascular disease (5.4\%), and previous cerebrovascular accident (7.4\%). Median preoperative left ventricular ejection fraction (LVEF) was $41.5 \%$ (IQR, 40.0-55.0\%). Almost two-thirds of the subjects presented in a critical preoperative state. The logistic EuroSCORE I was 29.3\% (IQR, 15.7-48.0\%). Surgical status was listed as emergency or salvage in most of the patients $(91.2 \%)$. In a slight majority of cases, VFWR was repaired on cardiopulmonary bypass (CPB). Median duration of CPB was 95.0 (IQR, 78.0-161.0) minutes and aortic cross-clamp (ACC) time was 49.5 (IQR, 37.0-88.0) minutes. Concomitant coronary artery bypass grafting (CABG) was performed in 19 patients (12.8\%), and VSR closure was associated with VFWR repair in 17 subjects $(11.5 \%)$. Mitral valve procedures were performed simultaneously in $4.7 \%$ of cases, and intraoperative cardiac assist device, either extracorporeal membrane oxygenation (ECMO) or ventricular assist device, was required in five individuals (3.4\%). Approximately one-quarter of patients had undergone a previous percutaneous coronary intervention, but only a minority had undergone previous CABG. IABP was inserted intraoperatively in 26 patients $(17.6 \%)$.

\section{Early outcomes}

Overall, in-hospital mortality was $31.1 \%(\mathrm{n}=46)$. Postoperative complications were common, including cardiac arrhythmia as the most frequent adverse event (20.9\%), followed by prolonged ( $>24$ hours) ventilation $(20.3 \%)$, acute kidney injury (16.9\%), perioperative myocardial infarction (MI) (12.2\%), pneumonia (8.1\%) and stroke $(2.7 \%)$. Re-thoracotomy for bleeding was also relatively common $(9.3 \%)$. Reoperation for other reasons was required in $4.1 \%$ of cases. The median length of hospital stay for the survivors was 11 (IQR, 7-20) days. Postoperative outcomes are depicted in Table 2.

Univariate analysis identified the associations between in-hospital mortality and older age $(\mathrm{P}=0.010)$, female sex $(\mathrm{P}<0.001)$, COPD $(\mathrm{P}=0.094)$, preoperative critical status $(\mathrm{P}<0.001)$, salvage surgery $(\mathrm{P}=0.053)$, logistic EuroSCORE I $(\mathrm{P}<0.001)$, New York Heart Association (NYHA) class IV $(\mathrm{P}=0.091)$, CPB time $(\mathrm{P}=0.044)$, ACC time $(\mathrm{P}=0.067)$, and intraoperative ECMO $(\mathrm{P}=0.089)$. Multivariable analysis identified only female sex [odds ratio (OR), 5.49; 95\% confidence interval $(\mathrm{CI}), 2.24-13.46 ; \mathrm{P}<0.001]$ and critical preoperative status (OR, 4.06; 95\% CI: 1.36-12.13; $\mathrm{P}=0.012$ ) as independent predictors of in-hospital mortality (Figure 1 and Table S3). A ROC curve was calculated for the logistic EuroSCORE I to determine the optimal cutoff value for predicting in-hospital mortality (Figure 2). The area under the curve (AUC) was 0.74 (95\% CI: 0.66-0.83; $\mathrm{P}<0.001)$. The optimal cut-off value identified was $33.6 \%$ with a sensitivity of $75 \%$ and a specificity of $71 \%$.

\section{Mid-term survival}

The median follow-up was 2.2 (IQR, 0.7-3.8) years. Figure 3 shows the Kaplan-Meier curve of overall survival (OS) for the whole population. The survival rates were $58.9 \%$ and $55.7 \%$ at three and five years, respectively. Among hospital survivors ( $\mathrm{n}=102)$, only $15(14.7 \%)$ subjects died during follow-up, with a three and five-year survival rate of $85.5 \%$ and $80.8 \%$, respectively (Figure 4). Figure 5 demonstrates a significant difference in the OS rates of all patients according to sex (log-rank, $\mathrm{P}=0.005)$, but not in the mortality rates of such patients among hospital survivors only $(\log$-rank, $\mathrm{P}=0.083)$.

\section{Discussion}

The main findings of the current study are as follows: (I) in-hospital mortality for post-AMI VFWR was $31.1 \%$; (II) 


\begin{tabular}{|c|c|c|c|c|}
\hline Variable & Total patients $(n=148)$ & Hospital survivors $(n=102)$ & Hospital non-survivors $(n=46)$ & $P$ value \\
\hline Age (years) & $66.5 \pm 11.1$ & $65.1 \pm 11.4$ & $69.8 \pm 9.9$ & 0.010 \\
\hline Sex (male) & $81(54.7)$ & $68(66.7)$ & $13(28.3)$ & $<0.001$ \\
\hline BMI $\left(\mathrm{kg} / \mathrm{m}^{2}\right)$ & $26.7 \pm 4.3$ & $26.6 \pm 3.9$ & $27.1 \pm 4.9$ & 0.545 \\
\hline Diabetes mellitus & 19 (12.8) & $16(15.7)$ & $3(6.5)$ & 0.123 \\
\hline COPD & $16(10.8)$ & $8(7.8)$ & $8(17.4)$ & 0.094 \\
\hline PVD & $8(5.4)$ & $6(5.9)$ & $2(4.3)$ & $>0.999$ \\
\hline Critical status & $93(62.8)$ & $54(52.9)$ & 39 (84.8) & $<0.001$ \\
\hline Unstable angina & $19(12.8)$ & $12(11.8)$ & $7(15.2)$ & 0.561 \\
\hline Emergent surgery & $75(50.7)$ & $55(53.9)$ & $20(43.5)$ & 0.240 \\
\hline Salvage surgery & $60(40.5)$ & $36(35.3)$ & $24(52.2)$ & 0.053 \\
\hline LVEF (\%) & $41.5(40.0-55.0)$ & $42.5(40.0-55.0)$ & $40.0(40.0-55.0)$ & 0.714 \\
\hline NYHA class IV & 59 (39.9) & $36(35.3)$ & $23(50.0)$ & 0.091 \\
\hline Logistic EuroSCORE I (\%) & $29.3(15.7-48.0)$ & $24.2(12.8-38.8)$ & $44.3(33.0-61.8)$ & $<0.001$ \\
\hline Previous CVA & $11(7.4)$ & $8(7.8)$ & $3(6.5)$ & 0.755 \\
\hline Previous PCI & 35 (23.6) & $27(26.5)$ & $8(17.4)$ & 0.229 \\
\hline Previous CABG & $7(4.7)$ & $5(4.9)$ & $2(4.3)$ & $>0.999$ \\
\hline Concomitant VSR & 17 (11.5) & $10(9.8)$ & 7 (15.2) & 0.339 \\
\hline Use of CPB & $83(56.1)$ & $56(54.9)$ & $27(58.7)$ & 0.667 \\
\hline CPB time (min) & $95.0(78.0-161.0)$ & $90.5(73.0-143.0)$ & $115.0(84.0-221.0)$ & 0.044 \\
\hline ACC & $67(45.3)$ & $46(45.1)$ & $21(45.7)$ & 0.950 \\
\hline ACC time (min) & 49.5 (37.0-88.0) & $46.0(34.0-65.0)$ & $70.0(39.0-151.0)$ & 0.067 \\
\hline Concomitant CABG & $19(12.8)$ & $13(12.7)$ & $6(13.0)$ & 0.960 \\
\hline Concomitant MVS & $7(4.7)$ & $3(2.9)$ & $4(8.7)$ & 0.204 \\
\hline Intra-operative IABP & $26(17.6)$ & $17(16.7)$ & $9(19.6)$ & 0.668 \\
\hline Intra-operative ECMO & $4(2.7)$ & $1(1.0)$ & $3(6.5)$ & 0.089 \\
\hline
\end{tabular}

Data are shown as mean \pm standard deviation or median (interquartile range), and number (\%), as appropriate. ACC, aortic cross-clamp; $\mathrm{BMI}$, body max index; CABG, coronary artery bypass grafting; COPD, chronic obstructive pulmonary disease; CPB, cardiopulmonary bypass; CVA, cerebrovascular accident; ECMO, extracorporeal membrane oxygenation; IABP, intra-aortic balloon pump; LVEF, left ventricular ejection fraction; MVS, mitral valve surgery; NYHA, New York Heart Association; PCI, percutaneous coronary intervention; PVD, peripheral vascular disease; VSR, ventricular septal rupture.

female sex and preoperative critical status were independent predictors of in-hospital mortality; (III) concomitant CABG during VFWR repair did not influence early survival; (IV) logistic EuroSCORE I may reliably predict mortality in this group of patients; $(\mathrm{V})$ beyond the early post-operative phase, mid-term survival is excellent.
VFWR is an uncommon but life-threatening mechanical complication of AMI. Despite significant improvements over the last two decades in overall mortality for patients with AMI, the outcome for patients who develop VFWR remains poor (1). Prompt diagnosis is key, and surgery, though challenging, is considered the treatment of choice $(9,10)$. 


\begin{tabular}{|c|c|}
\hline Variables & Total $(n=148)$ \\
\hline In-hospital mortality & $46(31.1)$ \\
\hline Length of stay* (days) & $11[7-20]$ \\
\hline Perioperative MI & $18(12.2)$ \\
\hline Pneumonia & $12(8.1)$ \\
\hline ARDS & $9(6.1)$ \\
\hline Stroke & $4(2.7)$ \\
\hline AKI & $25(16.9)$ \\
\hline Arrhythmia & $31(20.9)$ \\
\hline Gl complications & $6(4.1)$ \\
\hline Prolonged ventilation ( $>24$ hours) & $30(20.3)$ \\
\hline Readmission to ICU & $12(8.1)$ \\
\hline Re-thoracotomy for bleeding within 30 days & $18(12.2)$ \\
\hline \multicolumn{2}{|c|}{$\begin{array}{l}\text { Data are shown as median [interquartile range] or number (\%) } \\
\text { as appropriate. *, for hospital survivors. AKI, acute kidney injury; } \\
\text { ARDS, acute respiratory distress syndrome; GI, gastrointestinal; } \\
\text { ICU, intensive care unit; MI, myocardial infarction. }\end{array}$} \\
\hline
\end{tabular}

The present study examined data provided by the NHR to investigate outcomes in 148 patients with post-AMI VFWR who underwent surgical treatment from 2014 to 2019. Most of the previous studies regarding outcomes after VFWR repair are single-center experiences with small numbers of patients collected over a long period of time. The advantages of the current analysis lie in the fact that it includes all patients recently operated upon in the Netherlands during a six-year period and includes controlled in-hospital and post-discharge outcomes. The NHR is a national registry in which all cardiovascular interventions performed in the Netherlands are registered. A well-maintained nationwide registry has important clinical value in providing real-world data, in addition to controlled clinical trials that adhere to various exclusion criteria. Moreover, the completeness and accuracy of data input have improved since the inception of the NHR.

In our cohort, most of the patients were male (54.7\%), which contrasts to the previous knowledge that VFWR typically occurs in female subjects, above 60 years of age, as a complication of their first transmural MI in nonhypertrophic hearts (11). However, less than one-third of patients had undergone a previous revascularization procedure, either percutaneous or surgical. Major comorbidities and known cardiovascular risk factors, such as diabetes mellitus, peripheral vascular disease and previous cerebrovascular accidents were identified in a minority of patients and were not associated with higher inhospital mortality. In the CAUTION LVFWR $_{\text {study (3), which }}$ represents the largest report to date of patients undergoing cardiac surgery for post-AMI VFWR, the majority of subjects $(70 \%)$ were in cardiogenic shock at presentation, and cardiac arrest was identified as an independent risk factor for operative mortality. Similarly, in our cohort most patients presented in a critical status before surgery, and this condition was found to significantly increase the risk of death.

The role of CABG during VFWR repair remains controversial. Although earlier reports have shown a beneficial effect of concomitant CABG when indicated $(12,13)$, we did not find any early survival benefit of simultaneous CABG. We can hypothesize that the real effectiveness of the myocardial revascularization was underestimated by the low number of patients who underwent CABG (12.8\%). Indeed, in emergency situations, the execution of coronary angiography is not always possible due to the need to quickly proceed to surgery, and this intrinsically impacts the possibility to perform any angiography-guided revascularization. It is likely that concomitant CABG could be beneficial in patients with multivessel coronary artery disease (CAD), but not in patients that have single-vessel disease. In this cohort, less than $10 \%$ of patients were reported to have multivessel CAD, although $>50 \%$ of data were missing (results not shown). We believe that CABG should be applied, whenever possible, to reduce further ischemic risk associated with multivessel CAD by improving collateral flow to the myocardium. However, dedicated studies are warranted to specifically address the role of concomitant CABG in the setting of VFWR repair.

Short-term mechanical circulatory support (MCS) has emerged during recent years as a life-saving intervention in subjects with cardiogenic shock (14). Some authors have suggested a potential role of MCS in critical patients with VFWR $(15,16)$. These devices may be of help both to improve preoperative hemodynamic conditions, thereby allowing a safer bridge to surgical repair, and to provide a protected perioperative course (15). However, our results did not show any benefit of intraoperative MCS insertion for in-hospital survival. Such a result may be explained by many factors, including the low adoption rate $(n=5)$ 


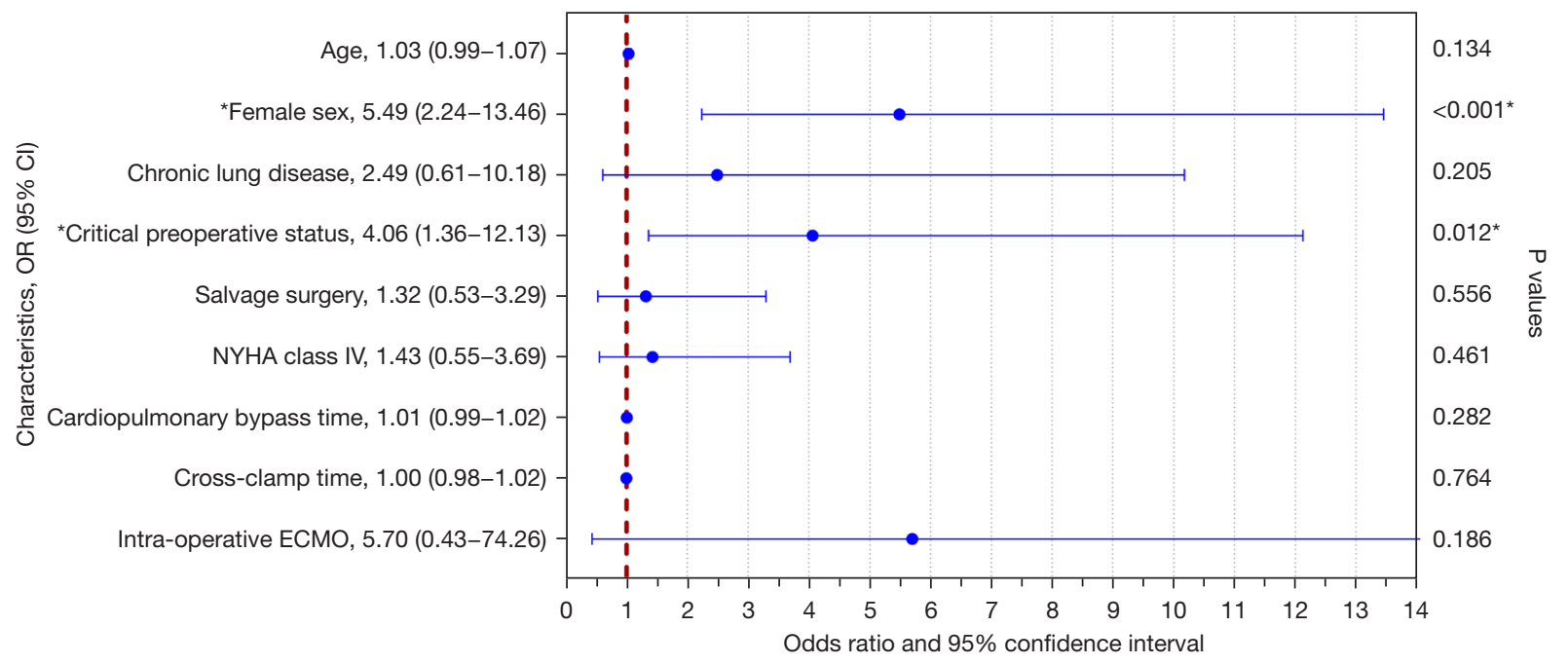

Figure 1 Forest plot of multivariable logistic regression analysis with odds ratios and $95 \%$ confidence intervals for in-hospital mortality after post-AMI VFWR. *, statistically significant $(\mathrm{P}<0.05)$. OR, odds ratio; CI, confidence interval; ECMO, extracorporeal membrane oxygenation; NYHA, New York Heart Association.

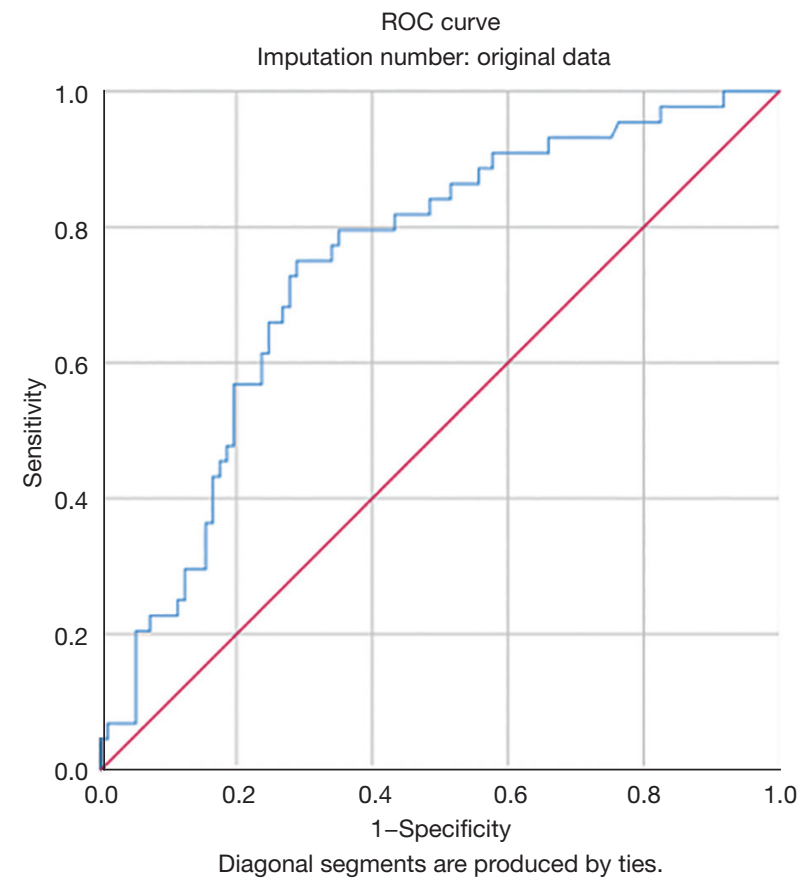

Figure 2 Receiver operating characteristic curve for the logistic EuroSCORE I as a predictor of in-hospital mortality in patients operated for post-AMI VFWR. ROC, receiver operating characteristic; AMI, acute myocardial infarction; VFWR, ventricular free-wall rupture.

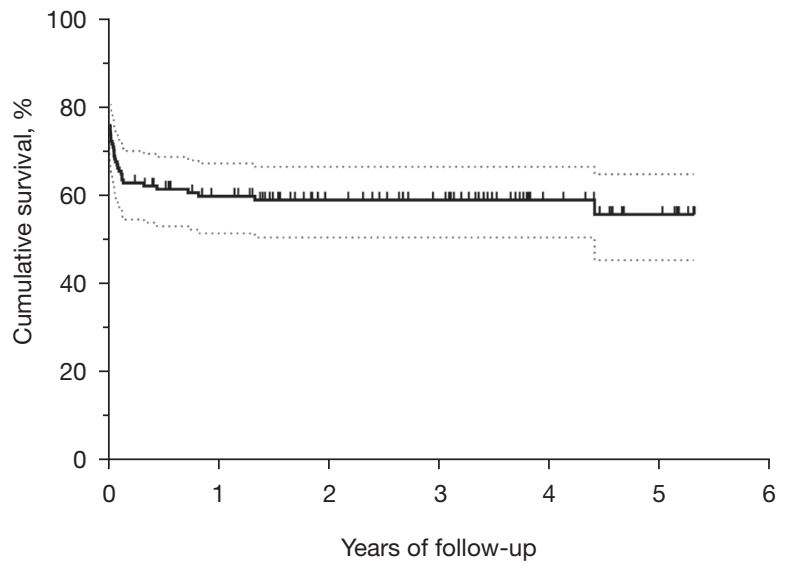

Pts. at risk

$$
\begin{array}{llllll}
148 & 74 & 53 & 43 & 22 & 11
\end{array}
$$

Figure 3 Kaplan-Meier survival curve of overall survival for all patients after surgical repair of post-AMI VFWR. AMI, acute myocardial infarction; VFWR, ventricular free-wall rupture.

and selection bias. On the other hand, lack of data on preoperative MCS use does not allow for consideration of the effect of these devices on clinical outcome.

We observed a high in-hospital mortality rate (almost one-third of the total patient population), which is consistent with the $17-34 \%$ operative mortality rate 


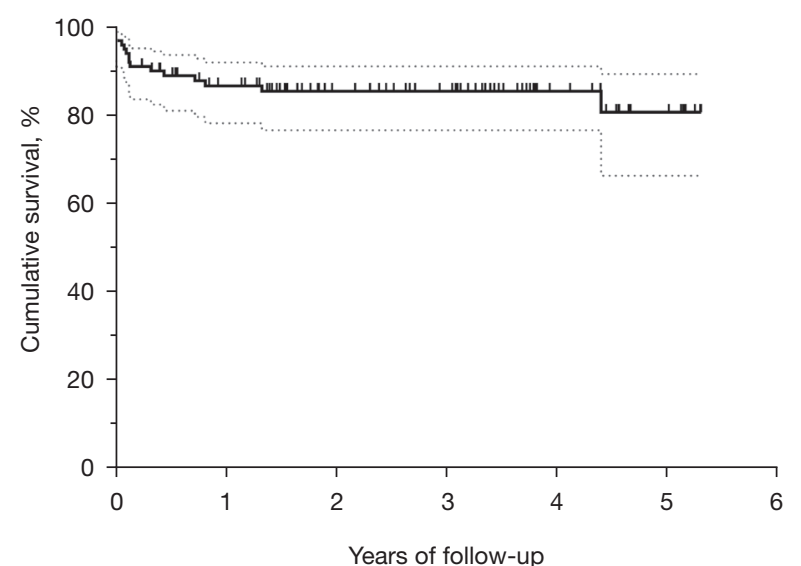

Pts. at risk

$\begin{array}{llllll}102 & 74 & 53 & 43 & 22 & 11\end{array}$

Figure 4 Kaplan-Meier survival curve of overall survival for hospital survivors after surgical repair of post-AMI VFWR. AMI, acute myocardial infarction; VFWR, ventricular free-wall rupture.

reported in the literature (4,5,17-19). In the present study, female sex was a strong independent predictor for unfavorable in-hospital outcome. This observation is in accordance with a previous multicenter study, involving 140 adults who underwent VFWR surgical repair, in which mortality varied significantly depending on gender (3). The underlying mechanisms by which female sex acts as a negative predictor in VFWR may reside in a difference in timing and type of MI presentation, and on many other poorly identified factors that would require further investigation. Interestingly, despite women being found to have a significantly poorer in-hospital outcome than men, mid-term survival data for hospital survivors showed an inverted trend between males and females, although this was not statistically significant.

The logistic EuroSCORE I was not included in the multivariable analysis, because it incorporates some of the variables already considered in our model (e.g., sex). However, in univariate analysis, in-hospital survivors had a significantly lower score than non-survivors. The logistic EuroSCORE I is an important risk-stratification model in cardiac surgery that was introduced in 1999 (20). In the past decade, the predictive power of this model has proven its value in clinical practice and quality monitoring, particularly in high-risk patient subgroups (21). In the current analysis, the median logistic EuroSCORE I was $29.3 \%$, which means that it only slightly underestimated the actual in-hospital mortality rate in this cohort $(31.1 \%)$. Although logistic
A

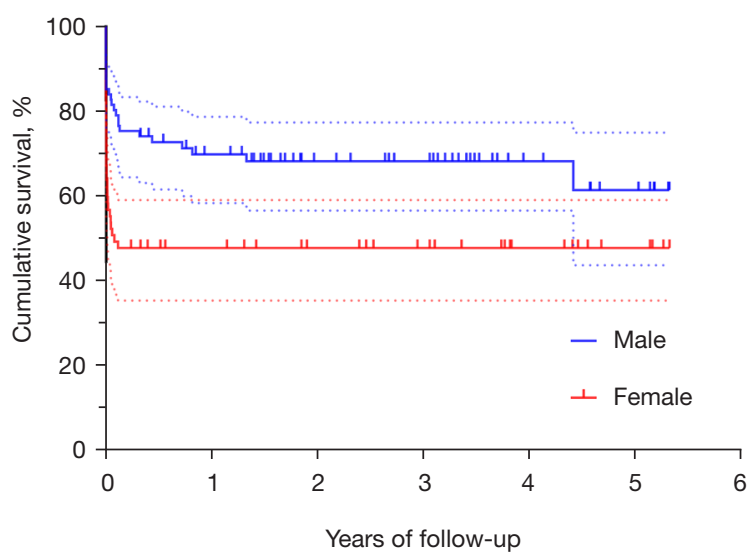

Pts. at risk

$\begin{array}{lllllll}\text { Male } & 81 & 47 & 32 & 26 & 12 & 7 \\ \text { Female } & 67 & 28 & 23 & 18 & 11 & 5\end{array}$

B

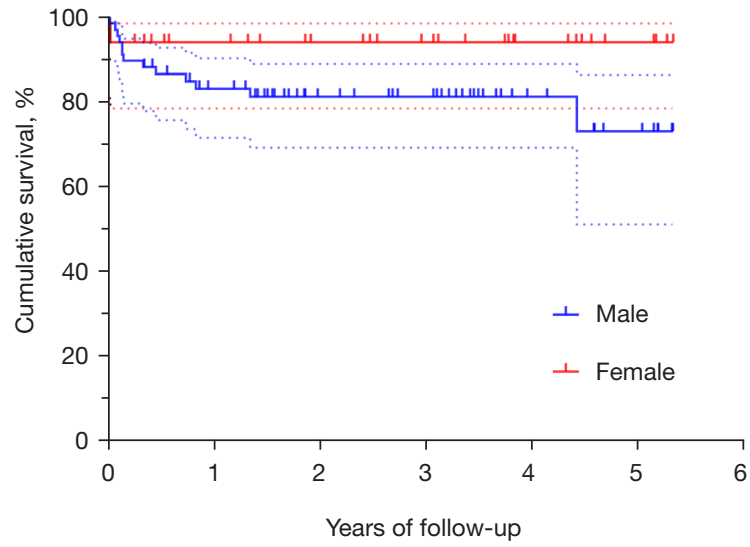

Pts. at risk

$\begin{array}{lllllll}\text { Male } & 68 & 47 & 32 & 26 & 12 & 7 \\ \text { Female } & 34 & 28 & 23 & 18 & 11 & 5\end{array}$

Figure 5 Kaplan-Meier survival curves of overall survival according to sex for all patients (A) and for hospital survivors (B) after surgical repair of post-AMI VFWR. AMI, acute myocardial infarction; VFWR, ventricular free-wall rupture.

EuroSCORE I was not specifically designed for patients undergoing surgery for post-AMI VFWR, this model can be reasonably used to predict early mortality in this setting. Indeed, ROC curve analysis showed that logistic EuroSCORE I had a good discriminatory power for inhospital mortality in VFWR patients, with an AUC of 0.74 (Figure 2). The optimal cut-off identified to select patients 
at higher risk of in-hospital mortality was $33.6 \%$.

In this study, the mean follow-up was 2.4 years, and the three-year and five-year OS rate was $59 \%$ and $55 \%$, respectively. Sakaguchi et al. reported a five-year survival rate of $74 \%$ and a mean follow-up of 2.4 years in a series of 32 patients with post-AMI VFWR (22). However, in their cohort, only eight patients required cardiopulmonary resuscitation at presentation, and hence the resulting inhospital mortality was only $15.6 \%$. Thus, their higher five-year survival compared with our findings may be due to a lower number of patients presenting with severe hemodynamic compromise requiring salvage surgery ( $25 \%$ vs. $40 \%$ ). However, in our study, it is interesting to highlight that when survival function is limited to hospital survivors only, the three-year survival rate was $85 \%$, and the five-year survival rate approached $81 \%$. Similar outcomes of a five-year survival rate of $81 \%$ for patients discharged from the hospital have recently been reported by Formica and colleagues (5). Therefore, this data suggests an expected favorable outcome for hospital survivors of such a high-risk surgery for post-AMI VFWR repair. In light of this, despite the high lethality of VFWR, which is frequently associated with an extremely severe clinical presentation, and the urgency and complexity of the primary intervention, early diagnosis and prompt surgery may play a key role in the management of this post-AMI complication and could provide an excellent life-expectancy for hospital survivors, which may possibly be comparable to the general noncomplicated MI population (23).

\section{Limitations}

Several limitations should be acknowledged. Firstly, because of the retrospective study design, we are unable to certify that all potential confounders have been examined. Indeed, the nationwide multicenter registry design necessitated a simplified data collection form with a limited number of variables to avoid missing data. Thus, the possibility that non-registered variables could have influenced the results of the multivariable analysis cannot be completely ruled out. For example, the database has no information on the interval between AMI and VFWR diagnosis, the time between VFWR recognition and surgical operation, the location and type of VFWR or the surgical technique used to repair the rupture. Secondly, large registry datasets depend heavily on accurate coding. The NHR perform regular audits and internal data checking. We have assumed that any residual coding errors are random and are therefore unlikely to render any bias. Thirdly, the number of patients enrolled may still be considered relatively small. Therefore, a prospective multicenter study incorporating a larger sample size would be useful to assess prognostic value of the risk factors identified. This study is further limited by lack of specific causes of mortality, and reasons for reoperation (e.g., ventricular re-rupture). Another limitation is the missing values of some variables. To minimize the effects of missing data, clinically relevant variables with up to $40 \%$ missing data were handled with multiple imputation.

\section{Conclusions}

The present nationwide study confirms that VFWR surgical repair is associated with high in-hospital mortality. However, mid-term survival in patients surviving the immediate postoperative period is encouraging. These observations emphasize the importance of prompt diagnosis and surgical management for subjects who develop VFWR after AMI.

\section{Acknowledgments}

On behalf of the Cardiothoracic Surgery Registration Committee of the Netherlands Heart Registration.

Funding: None.

\section{Footnote}

Conflicts of Interest: Prof. RL is a consultant for Medtronic, Getinge and LivaNova, and Member of the advisory board of Eurosets and Fresenius/Xenios. The other authors have no conflicts of interest to declare.

Open Access Statement: This is an Open Access article distributed in accordance with the Creative Commons Attribution-NonCommercial-NoDerivs 4.0 International License (CC BY-NC-ND 4.0), which permits the noncommercial replication and distribution of the article with the strict proviso that no changes or edits are made and the original work is properly cited (including links to both the formal publication through the relevant DOI and the license). See: https://creativecommons.org/licenses/by-nc-nd/4.0/.

\section{References}

1. Elbadawi A, Elgendy IY, Mahmoud K, et al. Temporal Trends and Outcomes of Mechanical Complications 
in Patients With Acute Myocardial Infarction. JACC

Cardiovasc Interv 2019;12:1825-36.

2. Blinc A, Noc M, Pohar B, et al. Subacute rupture of the left ventricular free wall after acute myocardial infarction. Three cases of long-term survival without emergency surgical repair. Chest 1996;109:565-7.

3. Matteucci M, Kowalewski M, De Bonis M, et al. Surgical Treatment of Post-Infarction Left Ventricular FreeWall Rupture: A Multicenter Study. Ann Thorac Surg 2021;112:1186-92.

4. Okamura H, Kimura N, Mieno M, et al. Sutureless repair for postinfarction left ventricular free wall rupture. J Thorac Cardiovasc Surg 2019;158:771-7.

5. Formica F, Mariani S, Singh G, et al. Postinfarction left ventricular free wall rupture: a 17-year single-centre experience. Eur J Cardiothorac Surg 2018;53:150-6.

6. Nederlandse Hart Registratie (NHR). Available online: http://www.nederlandehartregistratioe.nl/ (accessed Feb 2021).

7. Thygesen K, Alpert JS, Jaffe AS, et al. Third universal definition of myocardial infarction. Glob Heart 2012;7:275-95.

8. Jakobsen JC, Gluud C, Wetterslev J, et al. When and how should multiple imputation be used for handling missing data in randomised clinical trials - a practical guide with flowcharts. BMC Med Res Methodol 2017;17:162.

9. Matteucci M, Fina D, Jiritano F, et al. Treatment strategies for post-infarction left ventricular free-wall rupture. Eur Heart J Acute Cardiovasc Care 2019;8:379-87.

10. Matteucci M, Fina D, Jiritano F, et al. Sutured and sutureless repair of postinfarction left ventricular freewall rupture: a systematic review. Eur J Cardiothorac Surg 2019;56:840-8.

11. Slater J, Brown RJ, Antonelli TA, et al. Cardiogenic shock due to cardiac free-wall rupture or tamponade after acute myocardial infarction: a report from the SHOCK Trial Registry. Should we emergently revascularize occluded coronaries for cardiogenic shock? J Am Coll Cardiol 2000;36:1117-22.

12. Sutherland FW, Guell FJ, Pathi VL, et al. Postinfarction ventricular free wall rupture: strategies for diagnosis and treatment. Ann Thorac Surg 1996;61:1281-5.

13. Mantovani V, Vanoli D, Chelazzi P, et al. Post-infarction cardiac rupture: surgical treatment. Eur J Cardiothorac Surg 2002;22:777-80.

14. Stretch R, Sauer CM, Yuh DD, et al. National trends in the utilization of short-term mechanical circulatory support: incidence, outcomes, and cost analysis. J Am Coll Cardiol 2014;64:1407-15.

15. Formica F, Corti F, Avalli L, et al. ECMO support for the treatment of cardiogenic shock due to left ventricular free wall rupture. Interact Cardiovasc Thorac Surg 2005;4:30-2.

16. Matteucci M, Fina D, Jiritano F, et al. The use of extracorporeal membrane oxygenation in the setting of postinfarction mechanical complications: outcome analysis of the Extracorporeal Life Support Organization Registry. Interact Cardiovasc Thorac Surg 2020;31:369-74.

17. Kacer P, Adamkova V, Hubacek JA, et al. Post-infarction left ventricular free wall rupture: 12-years experience from the Cardiac Centre of the Institute of Clinical and Experimental Medicine in Prague, Czech Republic. Biomed Pap Med Fac Univ Palacky Olomouc Czech Repub 2020. [Epub ahead of print].

18. Haddadin S, Milano AD, Faggian G, et al. Surgical treatment of postinfarction left ventricular free wall rupture. J Card Surg 2009;24:624-31.

19. Matteucci M, Formica F, Kowalewski M, et al. Metaanalysis of surgical treatment for postinfarction left ventricular free-wall rupture. J Card Surg 2021;36:3326-33.

20. Nashef SA, Roques F, Michel P, et al. European system for cardiac operative risk evaluation (EuroSCORE). Eur J Cardiothorac Surg 1999;16:9-13.

21. Michel P, Roques F, Nashef SA, et al. Logistic or additive EuroSCORE for high-risk patients? Eur J Cardiothorac Surg 2003;23:684-7; discussion 687.

22. Sakaguchi G, Komiya T, Tamura N, et al. Surgical treatment for postinfarction left ventricular free wall rupture. Ann Thorac Surg 2008;85:1344-6.

23. Sulzgruber P, El-Hamid F, Koller L, et al. Long-term outcome and risk prediction in patients suffering acute myocardial infarction complicated by post-infarction cardiac rupture. Int J Cardiol 2017;227:399-403.

Cite this article as: Matteucci M, Ronco D, Ravaux JM, Massimi G, Di Mauro M, Houterman S, Maessen J, Beghi C, Severgnini P, Lorusso R; on behalf of the Cardiothoracic Surgery Registration Committee of the Netherlands Heart Registration. Surgical repair of post-infarction ventricular free-wall rupture in the Netherlands: data from a nationwide registry. Ann Cardiothorac Surg 2022;11(3):310-318. doi: 10.21037/acs-2021-ami-10 
Supplementary

Table S1 Clinical characteristics of patients with all variables reported before multiple imputation with valid percentages

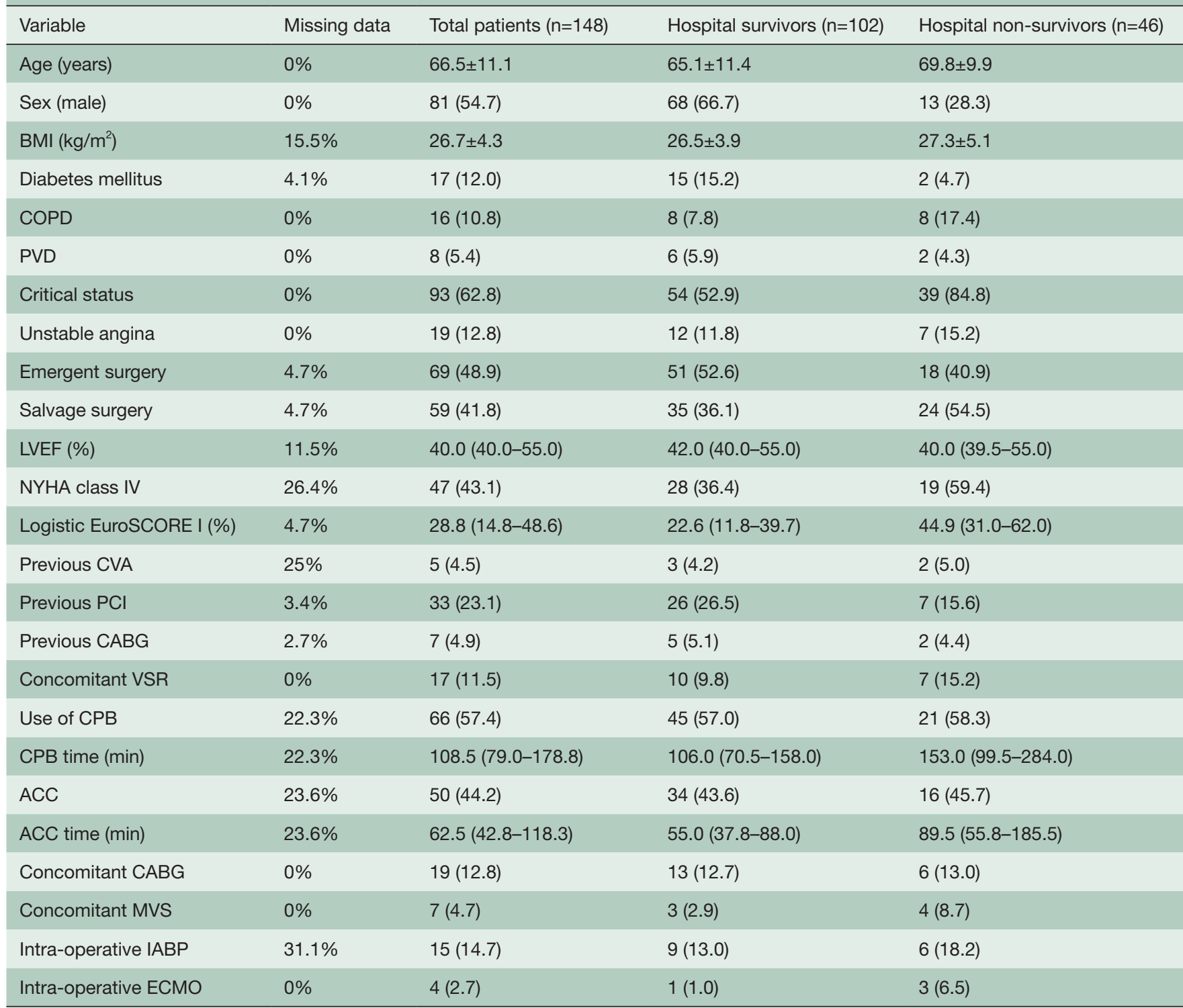

Data are shown as mean \pm standard deviation or median (interquartile range), and number (\%), as appropriate. ACC, aortic cross-clamp; $\mathrm{BMI}$, body max index; CABG, coronary artery bypass grafting; COPD, chronic obstructive pulmonary disease; CPB, cardiopulmonary bypass; CVA, cerebrovascular accident; ECMO, extracorporeal membrane oxygenation; IABP, intra-aortic balloon pump; LVEF, left ventricular ejection fraction; MVS, mitral valve surgery; NYHA, New York Heart Association; PCI, percutaneous coronary intervention; PVD, peripheral vascular disease; VSR, ventricular septal rupture. 


\begin{tabular}{lll}
\multicolumn{2}{l}{ Table S2 Postoperative in-hospital outcomes of patients with all variables reported before multiple imputation with valid percentages } \\
\hline Variable & Missing data & Total patients $(\mathrm{n}=148)$ \\
\hline In-hospital mortality & $0 \%$ & $46(31.1)$ \\
\hline Length of stay* (days) & $3.9 \%$ & $11.0(6.8-20.0)$ \\
\hline Perioperative MI & $23.6 \%$ & $11(9.7)$ \\
\hline Pneumonia & $8.8 \%$ & $9(6.7)$ \\
ARDS & $10.1 \%$ & $6(4.5)$ \\
Stroke & $5.4 \%$ & $3(2.1)$ \\
\hline AKI & $6.1 \%$ & $23(16.5)$ \\
Arrhythmia & $4.7 \%$ & $29(20.6)$ \\
GI complication & $4.7 \%$ & $5(3.5)$ \\
Prolonged ventilation $(>24$ hours) & $5.4 \%$ & $28(20.0)$ \\
\hline Readmission to ICU & $10.8 \%$ & $9(6.8)$ \\
\hline Re-thoracotomy for bleeding within 30 days & $27.0 \%$ & $10(9.3)$ \\
\hline
\end{tabular}

Data are shown as median (interquartile range) or number (\%), as appropriate; *, for hospital survivors. AKI, acute kidney injury; ARDS, acute respiratory distress syndrome; GI, gastrointestinal; ICU, intensive care unit; MI, myocardial infarction.

Table S3 Detailed results of multivariable logistic regression analysis

\begin{tabular}{llll}
\hline Variable & Odds ratio & $95 \%$ confidence interval & P value \\
\hline Age & 1.03 & $0.99-1.07$ & 0.134 \\
\hline Female sex & 5.49 & $2.24-13.46$ & $0.001^{*}$ \\
\hline COPD & 2.49 & $0.61-10.18$ & 0.205 \\
\hline Critical status & 4.06 & $1.36-12.13$ & $0.012^{*}$ \\
\hline Salvage surgery & 1.32 & $0.53-3.30$ & 0.556 \\
\hline NYHA class IV & 1.43 & $0.55-3.69$ & 0.461 \\
\hline CPB time & 1.01 & $0.995-1.02$ & 0.282 \\
\hline ACC time & 0.997 & $0.98-1.02$ & 0.764 \\
\hline Intra-operative ECMO & 5.70 & $0.43-74.26$ & 0.186 \\
\hline
\end{tabular}

*, statistically significant $(\mathrm{P}<0.05)$. ACC, aortic cross-clamp; COPD, chronic obstructive pulmonary disease; CPB, cardio-pulmonary bypass; ECMO, extracorporeal membrane oxygenation; NYHA, New York Heart Association. 